\title{
Perfil nutricional dos indígenas Xukuru-Kariri, Minas Gerais, de acordo com diferentes indicadores antropométricos e de composição corporal
}

\author{
Nutritional profile of the Xukuru-Kariri indigenous people \\ in the state of Minas Gerais in accordance with different \\ anthropometric and body composition indicators
}

Bárbara dos Santos Simões ${ }^{1}$

George Luiz Lins Machado-Coelho ${ }^{2}$

João Luiz Pena ${ }^{3}$

Silvia Nascimento de Freitas ${ }^{1}$

${ }^{1}$ Departamento de Nutrição Clínica e Social, Escola de Nutrição, Universidade Federal de Ouro Preto. Rua Diogo de Vasconcelos 122. 35400-000 Ouro Preto MG.

barbarassimoes@gmail.com ${ }^{2}$ Departamento de Ciências Médicas, Escola de Farmácia, Universidade Federal de Ouro Preto.

3 Departamento de Engenharia Sanitária e Ambiental, Universidade Federal de Minas Gerais

\begin{abstract}
The scope of this study was to evaluate the nutritional profile of indigenous XukuruKariri villagers in the state of Minas Gerais between seven and seventy-eight years of age in accordance with the different anthropometric and body composition indicator. The measurements were: weight, height, waist circumference (WC) and body fat percentage (BF\%). The sensitivity and specificity of anthropometric indices were calculated with a confidence interval of $95 \%$ and positive predictive value (PPV) and negative predictive value $(N P V)$. A total of 58 individuals was evaluated, of which $56.9 \%(n=33)$ were male and $43.1 \%$ ( $n=25)$ were female. Specificity was greater than sensitivity in all indices. It was seen that $29 \%$ of individuals were classified as having excess body fat as assessed by BMI and hand-tohand bioimpedance and 50\% of subjects had high body fat in relation to BMI and WC. Studies need to be conducted with different ethnic groups in order to construct indicators for specific nutritional guidance of indigenous health services.
\end{abstract}

Key words Indigenous health, Sensitivity and specificity, Anthropometrics
Resumo O objetivo deste artigo foi avaliar o perfil nutricional dos indígenas Xukuru-Kariri entre 7 anos e 78 anos aldeados em Minas Gerais de acordo com os diferentes indicadores antropométricos e de composição corporal. As medidas aferidas foram: peso, estatura, circunferência da cintura (CC) e percentual de gordura corporal (\%GC). Foram calculadas a sensibilidade e a especificidade para os indices antropométricos com intervalo de confiança de 95\% e o valor preditivo positivo $(V P P) e$ negativo (VPN). A população foi constituída por 58 indivíduos, sendo $56,9 \%(n=33)$ do sexo masculino e $43,1 \%(n=25)$ do sexo feminino. A especificidade foi superior à sensibilidade em relação a todos os indices. Observou-se que 29\% dos indivíduos foram classificados com excesso de adiposidade corporal quando avaliados pelo IMC e pela bioimpedância mão a mão e 50\% dos indivíduos apresentaram alta adiposidade corporal em relação ao IMC e a CC. É necessário que sejam realizados estudos com diferentes etnias a fim de se construir indicadores nutricionais específicos para orientação de serviços de saúde indígenas.

Palavras-chave Saúde indígena, Sensibilidade e especificidade, Antropometria 


\section{Introdução}

Atualmente, vivem no Brasil aproximadamente 800 mil índios, o que corresponde a cerca de $0,4 \%$ da população brasileira. Eles estão distribuídos em 683 Terras Indígenas e algumas áreas urbanas ${ }^{1}$. Devido às relações de contato interétnicos com a sociedade, esses indivíduos vêm passando por um acelerado e crescente processo de mudanças socioculturais que podem influenciar diretamente seus hábitos alimentares e consequentemente refletirem mudanças no estado nutricional ${ }^{2}$.

No plano individual, o estado nutricional é resultado do equilíbrio entre o consumo de nutrientes e o gasto energético do organismo para suprir as necessidades biológicas ${ }^{3}$. Ele também expressa uma dimensão histórica e social em que há relações entre homem-natureza-alimento que se estabelece no interior de uma determinada sociedade $^{4,5}$.

Ao se considerar a interação dos indígenas com outros grupos étnicos, observa-se a sua vulnerabilidade a distúrbios nutricionais, que compreende desde a desnutrição energético-protéica até graus avançados de obesidade ${ }^{2}$. No entanto, há escassez de estudos sobre o estado nutricional desses grupos étnicos ${ }^{2,6,7}$, e associado a este fato, há limitação de pesquisas que definam quais os indicadores antropométricos e de composição corporal são mais apropriados para a avaliação destes indivíduos.

A Organização Mundial de Saúde (OMS) recomenda que sejam realizados estudos específicos para diferentes grupos étnicos com a finalidade de se estabelecer padrões de referência que permitam avaliar as condições de saúde e nutrição destes indivíduos ${ }^{8}$.

Em estudos epidemiológicos os indicadores antropométricos comumente utilizados para se identificar o estado nutricional são o índice de massa corporal (IMC), circunferências da cintura (CC), relação cintura-quadril, braquial e medidas de dobras cutâneas 9 . Apesar de alguns autores realizarem estudos com diferentes grupos populacionais, atualmente os indicadores nutricionais tem como referência populações não indígenas ${ }^{10-12}$. Sabe-se que, esses povos apresentam características étnicas, sociais e culturais diferentes e muitas vezes são avaliados segundo parâmetros de outras populações de modo que tais valores podem não refletir o seu verdadeiro estado nutricional ${ }^{13}$. Dessa forma, é necessário que haja mais estudos com esse grupo para que se possam estabelecer critérios e indicadores que reflitam de forma mais fidedigna a saúde e o estado nutricional desses indivíduos.
Importante também ressaltar que o IMC tem sido recomendado pela OMS e utilizado em estudos epidemiológicos como um indicador da gordura corporal $^{14-17}$. Entretanto, alguns trabalhos têm questionado seu uso como objetivo de diagnosticar a gordura corporal em diferentes faixas etárias, uma vez que o IMC não consegue distinguir gordura corporal da massa magra ${ }^{18-20}$.

Assim, este artigo tem como objetivo avaliar o estado nutricional dos indígenas Xukuru-Kariri aldeados em Minas Gerais de acordo com os diferentes indicadores antropométricos e de composição corporal.

\section{Materiais e métodos}

\section{Desenho e população do estudo}

Estudo epidemiológico de delineamento transversal foi realizado na Terra Indígena XukuruKariri, localizada no município de Caldas, Minas Gerais no ano de 2009. A avaliação nutricional foi realizada no universo populacional. Foram excluídas dessa análise os indivíduos menores de 7 anos, as gestantes e aqueles que não seguiram as instruções pré-estabelecidas para a realização da bioimpedância.

\section{Avaliação nutricional}

Para a determinação do diagnóstico do estado nutricional, as medidas aferidas foram: peso, estatura, CC e percentual de gordura corporal (\%GC).

O peso foi mensurado utilizando balança eletrônica digital, marca Tanita ${ }^{\circledR}$, com capacidade para $150 \mathrm{Kg}$ e a aferição da altura foi realizada com o estadiômetro Alturexata ${ }^{\circledR}$ de altura máxima de 2,13m. A partir do cálculo do IMC, os indivíduos de 7 a 19 anos foram comparados com os padrões de escore $z$ de IMC/idade segundo a $\mathrm{OMS}^{21}$. Os adultos (20 a 59 anos) foram classificados de acordo com os pontos de corte propostos pela $\mathrm{OMS}^{22}$ e os idosos (maior ou igual a 60 anos de idade), por aqueles de acordo com Lipschit ${ }^{23}$. Posteriormente, os indivíduos foram agrupados em duas categorias: sem excesso de peso corporal, aqueles que apresentavam baixo peso ou eutrofia e com excesso de peso corporal, os que estavam com sobrepeso ou obesidade.

A CC foi obtida durante a expiração normal com fita métrica inelástica com extensão máxima de 2 metros, sendo circundada a circunferência horizontal localizada no ponto médio entre o 
último arco costal e a crista ilíaca. Os indivíduos foram classificados de acordo os pontos de corte propostos pela International Diabetes Federation $^{24}$ (IDF), aqueles com idade inferior a 20 anos foram considerados com excesso de adiposidade central, quando estavam acima do percentil 90, de acordo com a população de referência para crianças e adolescentes americanas com descendência européia. Os adultos e idosos, foram classificados com excesso de adiposidade central quando possuíam valores de CC iguais ou maiores a $90 \mathrm{~cm}$ para os homens e $80 \mathrm{~cm}$ para as mulheres ${ }^{10}$.

Já o \%GC, foi estimado utilizando-se a bioimpedância bipolar pé a pé através da balança Tanita $^{\circledR}$, bioimpedância mão a mão pelo Monitor de Gordura Corporal Omron BF 330 e a bioimpedância tetrapolar que foi realizada com os aparelhos Maltron (tetrapolar 1) e Byodinamics modelo 310e (tetrapolar 2). Na classificação dos indivíduos menores de 20 anos foram utilizados os pontos de corte propostos por Taylor et al. ${ }^{11}$ para os indivíduos com idade igual ou superior a 20 anos, os propostos por Gallagher et al. ${ }^{12}$.

\section{Análise estatística}

Para a determinação do escore z IMC/ idade foi utilizado o programa da World Health Organization - Anthroplus, versão 2.0.2. As análises dos dados foram realizadas no programa Predictive Analytics Software Statistics (PASW, versão 17.0). Foi utilizado o Programa OpenEpi, versão 2.3.1 para o cálculo da sensibilidade, especificidade, valor preditivo positivo (VPP) e negativo (VPN) dos métodos antropométricos em relação ao método de referência (IMC).

\section{Comitê de ética}

A pesquisa foi submetida às lideranças indígenas, ao Conselho Local de Saúde, ao Comitê de Ética em Pesquisa da Universidade Federal de Ouro Preto (CEP-UFOP), ao Comitê Nacional de Ética em Pesquisa (CONEP) e à Fundação Nacional do Índio (Funai), com a finalidade de obter a autorização de ingresso na Terra Indígena Xukuru-Kariri. Antes de iniciar a coleta de dados na terra indígena, o coordenador e os responsáveis pelo projeto, visitaram o local, explicaram o trabalho que seria desenvolvido e solicitaram às lideranças da comunidade o consentimento escrito para realização da mesma. A pesquisa foi financiada pela Fundação de Amparo à Pesquisa de Minas Gerais (FAPEMIG) e Conselho Nacional de Desenvolvimento Científico e Tecnológico (CNPq).

\section{Resultados}

A população de estudo foi constituída por 58 indivíduos, sendo 56,9\% ( $\mathrm{n}=33)$ do sexo masculino e $43,1 \%(n=25)$ do feminino. A idade média foi de 21,7 anos, sendo que o indivíduo mais novo tinha 7,2 anos e o mais velho 77,9 anos. Quanto aos indicadores antropométricos, a distribuição dos valores médios de acordo com as categorias criança/adolescente, adulto e idoso se encontra na Tabela 1.

De acordo com a classificação do IMC, observou-se que apenas $1,7 \%(\mathrm{n}=1)$ dos indivíduos apresentaram baixo IMC, 65,5 $(\mathrm{n}=38)$ eram eutróficos, mas 27,6\% $(\mathrm{n}=16)$ apresentavam sobrepeso e $5,2 \%(n=3)$ obesidade. Em relação

Tabela 1. Indicadores antropométricos para a população indígena Xukuru-Kariri; Caldas, 2009

\begin{tabular}{lccc}
\hline \multicolumn{1}{c}{ Indicadores } & $\begin{array}{c}\text { Criança/Adolescente } \\
\text { Média } \pm \mathbf{D P}(\mathbf{N})\end{array}$ & $\begin{array}{c}\text { Adulto } \\
\text { Média }(\mathbf{c m}) \pm \mathbf{D P}(\mathbf{N})\end{array}$ & $\begin{array}{c}\text { Idoso } \\
\text { Média } \pm \mathbf{D P}(\mathbf{N})\end{array}$ \\
\hline IMC $\left(\mathrm{kg} / \mathrm{m}^{2}\right)$ & $19,92 \pm 5,67(26)$ & $24,57 \pm 2,81(30)$ & $23,54 \pm 2,22(2)$ \\
CC $(\mathrm{cm})$ & $66,41 \pm 12,20(25)$ & $81,56 \pm 7,84(29)$ & $84,60 \pm 1,27(2)$ \\
\% GC pé a pé & $20,11 \pm 9,36(25)$ & $23,18 \pm 9,14(28)$ & $28,05 \pm 8,27(2)$ \\
\% GC mão a mão & $22,33 \pm 8,98(13)$ & $20,83 \pm 9,22(27)$ & $33,25 \pm 9,68(2)$ \\
\% GC Tetrapolar 1 & $20,35 \pm 8,84(23)$ & $23,70 \pm 9,28(27)$ & $30,18 \pm 5,19(2)$ \\
\% GC Tetrapolar 2 & $18,97 \pm 8,28(22)$ & $21,73 \pm 8,76(27)$ & $30,20 \pm 5,93(2)$ \\
\hline
\end{tabular}

IMC: Índice de massa corporal $\left(\mathrm{kg} / \mathrm{m}^{2}\right)$. CC: circunferência da cintura. \% GC pé a pé: percentual de gordura corporal de acordo com a Tanita ${ }^{\circledR}$. \% GC mão a mão: percentual de gordura corporal de acordo com o Omron. \% GC Tetrapolar 1: percentual de gordura corporal de acordo com Maltron. \% GC Tetrapolar 2: percentual de gordura corporal de acordo com o Byodinamics. 
à circunferência de cintura 21,4\% (n = 12) apresentaram excesso de adiposidade central.

As prevalências do excesso de adiposidade corporal foram avaliadas pelo \%GC de acordo com a bioimpedância pé a pé $(\mathrm{n}=55)$, mão a mão $(\mathrm{n}=42)$ e bioimpedância Tetrapolar $(\mathrm{n}=$ 52) e Tetrapolar $2(n=51)$. Observou-se excesso de adiposidade corporal em 25\% $(\mathrm{n}=13), 19 \%$ $(\mathrm{n}=8), 18,2 \%(\mathrm{n}=10), 17,6 \%(\mathrm{n}=9)$ dos indivíduos de acordo com o Tetrapolar 1, mão a mão, bioimpedância pé a pé, e Tetrapolar 2, respectivamente.

A Tabela 2 descreve os valores de sensibilidade, especificidade, VPP e VPN dos métodos em relação ao IMC.

Assumindo o IMC como método de referência, a especificidade foi superior à sensibilidade em relação a todos os índices. O método que apresentou menor sensibilidade e especificidade foi o \%GC mão a mão, em que apenas 29\% dos indivíduos foram classificados corretamente com excesso de adiposidade corporal. $\mathrm{O}$ maior valor de sensibilidade foi obtido pela medida da CC em que $50 \%$ dos indivíduos foram classificados corretamente com alta adiposidade corporal, apresentando este método uma especificidade de $92 \%$.

\section{Discussão}

A prevalência de excesso de peso variou de acordo com o índice utilizado. Em nosso estudo, $32,8 \%$ dos indivíduos apresentaram excesso de peso quando avaliados pelo IMC, $21 \%$ pela circunferência da cintura, 25\% pelo Tetrapolar 1, $19 \%$ pela bioimpedância mão a mão, 18,2\% pela bioimpedância pé a pé e $17,6 \%$ pelo Tetrapolar 2 .
Castro et al. ${ }^{6}$ observaram entre os indígenas Kaingáng no Rio Grande do Sul, que 8,9\% apresentavam excesso de peso e/ou obesidade de acordo com o IMC. Gugelmin e Santos ${ }^{7}$ constataram na Terra Indígena Sangra-Douro-Volta Grande, Mato Grosso, que 78\% dos adultos tinham IMC elevado e atribuíram seus achados de sobrepeso e obesidade à transição cultural e alimentar a que essas populações foram submetidas ao longo do tempo. Já Almeida et al. ${ }^{25}$ não observaram em indígenas Xukuru-Kariri residentes na Fazenda do Canto, Alagoas presença de desvios nutricionais. Dessa forma, o excesso de peso entre os indígenas Xukuru-Kariri aldeados em Minas Gerais, pode ser explicado devido ao processo de modificação de seus hábitos alimentares a qual esses indivíduos foram submetidos há aproximadamente 18 anos, devido a sua migração do estado de Alagoas para Minas Gerais ${ }^{26}$.

Dentre os fatores possivelmente associados às diferentes prevalências encontradas entre diferentes etnias, deve-se pontuar tanto o método utilizado, quanto suas limitações. Sabe-se que apesar do IMC ser muito utilizado na avaliação do estado nutricional de populações, este não permite diferenciar massa magra de gordura corporal. Outro fator relevante a ser mencionado é que no Brasil, estudos com populações indígenas adotam os pontos de cortes utilizados para população em geral, o que dificulta ainda mais a avaliação de diferentes grupos étnicos, como o estudado no presente trabalho. Pan et al. ${ }^{27}$ e Woo et al..$^{28}$ propuseram pontos de cortes de IMC mais baixos, em torno de $27 \mathrm{~kg} / \mathrm{m}^{2}$, para diagnóstico de obesidade para população asiática. No Brasil, os pontos de cortes utilizados para IMC são tidos como referência para populações não asiáticas,

Tabela 2. Sensibilidade, especificidade e valor preditivo positivo (VPP) e negativo (VPN) dos métodos diagnósticos de excesso de adiposidade corporal em relação ao método de referência (IMC) para a população indígena Xukuru-Kariri; Caldas, 2009.

\begin{tabular}{lcccr}
\hline \multicolumn{1}{c}{ Indicadores } & Sensibilidade & Especificidade & VPP & VPN \\
\hline CC & 0,50 & 0,92 & 0,75 & 0,79 \\
\% GC pé a pé & 0,41 & 0,92 & 0,70 & 0,77 \\
\% GC mão a mão & 0,29 & 0,88 & 0,62 & 0,64 \\
\% GC Tetrapolar 1 & 0,41 & 0,88 & 0,63 & 0,75 \\
\% GC Tetrapolar 2 & 0,35 & 0,91 & 0,66 & 0,73
\end{tabular}

CC: circunferência da cintura. \%GC pé a pé: percentual de gordura corporal de acordo com a Tanita ${ }^{\circledR}$ \%GC mão a mão: percentual de gordura corporal de acordo com o Omron. \% GC Tetrapolar 1: percentual de gordura corporal de acordo com Maltron. \% GC Tetrapolar 2: percentual de gordura corporal de acordo com o Byodinamics. VPP:Valor preditivo positivo. VPN: Valor preditivo negativo. 
porém, devido a semelhanças étnicas entre indígenas e asiáticos, tais parâmetros poderiam ser mais adequados para a população em questão ${ }^{29}$.

Outros indicadores relevantes a serem mencionados são o \%GC e a adiposidade central, que quando em excesso, têm sido utilizados como preditores de doenças crônicas. O primeiro indicador necessita de aparelhos de bioimpedância mais sofisticados, ao passo que o segundo pode ser avaliado pela CC, sendo facilmente aferida em grupos populacionais ${ }^{10,15}$. Lourenço ${ }^{30} \mathrm{em}$ uma população adulta indígena de Suruí, Rondônia observaram que 23,2\% dos indivíduos apresentavam excesso de adiposidade corporal quando avaliados de acordo com a Tanita ${ }^{\circledR}$ Já para a adiposidade central, Gimeno et al. ${ }^{2}$ constataram que dos adultos indígenas Aruak, 52\% apresentavam excesso utilizando a CC

Neste estudo, os valores de especificidade foram elevados para todos os indicadores em relação ao IMC; já a CC foi a que apresentou maior sensibilidade, isto é, maior número de verdadeiros positivos e maior capacidade de identificar os indivíduos que apresentavam desvio nutricional quando comparado com o método de referência (IMC). Em uma pesquisa com adultos nos Estados Unidos comparou-se o IMC com a bioimpedância tetrapolar e foi observada uma sensibilidade de $86 \%$ e especificidade de $72 \%{ }^{31}$. Valores semelhantes foram encontrados por Cândido et al. ${ }^{32}$ que verificaram uma sensibilidade de $100 \%$ entre IMC e biompedância tetrapolar utilizando pontos de cortes de $25 \%$ e $35 \%$ para adolescentes do sexo masculino e feminino, respectivamente. Freitas et al..$^{33}$ observaram em adultos uma sensibilidade de 90,3\% entre IMC e bioimpedância bipolar pé a pé. Tais valores diferem dos encontrados em nosso estudo, o motivo para essa diferença pode estar na definição dos pontos de corte adotados como critério de referência e principalmente devido à diferente etnia avaliada.

Uma limitação para o uso dos aparelhos de bioimpedância é que as equações utilizadas pelos fabricantes são elaboradas a partir de amostras generalizadas ou específicas, podendo não reproduzir os mesmos resultados obtidos em populações com características diferentes daquelas que primariamente foram validadas ${ }^{34}$. Portanto, apesar da realização de estudos com enfoque no desenvolvimento e validação de equações de regressão preditivas, generalizadas ou especificas, ade- quadas para a estimativa dos componentes da composição corporal por meio da bioimpedância em diferentes populações terem sido realizadas nos últimos anos, essas avaliações carecem para populações indígenas ${ }^{35-37}$. Pois tais pesquisas são importantes para melhorar a exatidão e a confiabilidade desta técnica de predição, de forma a ser utilizada como ferramenta para monitorar o estado nutricional dos indivíduos.

Apesar de diversos estudos que descrevem o estado nutricional de indígenas terem crescido nas últimas décadas, poucos são os que analisam todos os seguimentos da população e como não há referências específicas para esses indivíduos, tais resultados podem não corresponder ao estado nutricional real dos mesmos ${ }^{7,34,38}$.

Os resultados deste trabalho demonstraram que o uso da CC, juntamente com o IMC contribuem na identificação correta dos indivíduos com excesso de gordura corporal. Vale a pena ressaltar que a prevalência dos desvios nutricionais é dependente dos métodos utilizados. Deve-se considerar que ao se utilizar um método com alta sensibilidade, implicará na classificação de desvios nutricionais de um maior número de pessoas, consequentemente com maior frequência de verdadeiros falsos positivos (VFP), diminuindo a especificidade. No entanto, o uso simultâneo do IMC com a CC terá um ganho na confiabilidade, pois o excesso de sensibilidade do IMC é controlado pela especificidade do $\mathrm{CC}^{39}$.

O presente estudo apresentou algumas limitações metodológicas para o quesito sensibilidade/especificidade, como exemplo, o tamanho amostral e a deficiência de estudos de validação de indicadores antropométricos de populações indígenas, os quais permitiriam o estudo comparativo dos resultados observados. No entanto, apesar dos numerosos métodos de avaliação da composição corporal disponíveis na literatura, poucos são os estudos que avaliam a reprodutibilidade e a acurácia dos mesmos, principalmente entre a população indígena. Dessa forma, propõe-se que sejam realizados estudos com diferentes etnias, pois considerando a diversidade étnica que os caracteriza e a transição epidemiológica aos quais esses povos vêm passando, a construção de indicadores nutricionais específicos poderá refletir na orientação, organização, planejamento e melhoria da qualidade dos serviços de saúde específicos para os mesmos. 


\section{Colaboradores}

BS Simões participou da revisão de literatura, coleta, processamento e análise estatística do banco de dados e redigiu o artigo. JL Pena participou na coleta de dados e como facilitador do contato com os indígenas; SN Freitas e GLL Machado-Coelho elaboraram o projeto e delineamento da pesquisa, interpretação dos dados, discussão dos resultados, redação e aprovação da versão final. Todos os autores participaram da redação do manuscrito e aprovaram a sua versão final.

\section{Agradecimentos}

Agradecemos aos indígenas Xukuru-Kariri pela disponibilidade, aos acadêmicos de Nutrição e Medicina da Universidade Federal de Ouro Preto pela contribuição durante a coleta de dados, a Fundação de Amparo à Pesquisa de Minas Gerais, ao Conselho Nacional de Desenvolvimento Científico e Tecnológico pela concessão da bolsa de mestrado e financiamento desta pesquisa.

\section{Referências}

1. Brasil. Fundação Nacional do Índio. [site da Internet] [acessado 2011 jul. 12]. Disponível em: http:// www.funai.gov.br

2. Gimeno SGA, Rodrigues D, Pagliaro H, Cano EM, Lima EES, Baruzzi RG. Perfil metabólico e antropométrico de índios Aruák: Mehináku, Waurá e Yawalapíti, Alto Xingu, Brasil Central, 2000/2002. Cad Saude Publica 2007; 23(8):1946-1954.

3. Brasil. Ministério da Saúde. Vigilância alimentar e nutricional - Sisvan: orientações básicas para a coleta, processamento, análise de dados e informação em serviços de saúde. [site da Internet] [acessado 2011 mar 25]. Disponível em: http://bvsms.saude. gov.br/bvs/publicacoes/orientacoes_basicas_sisvan. pdf

4. Vasconcelos FAG. Avaliação nutricional de coletividades. 4a Edição. Florianópolis: UFSC; 2008.

5. Mello ED. O que significa a avaliação do estado nutricional. J Pediatr 2002; 78(5):357-358.

6. Castro, TG, Schuch I, Conde WL, Veiga J, Leite MS, Dutra CLC, Zuchinali P, Barufaldi LA. Estado nutricional dos indígenas Kaingáng matriculados em escolas indígenas do estado do Rio Grande do Sul, Brasil. Cad Saude Publica 2010; 26(9):1766-1776.

7. Gugelmin SA, Santos RV. Uso do índice de massa corporal na avaliação do estado nutricional de adultos indígenas Xavante, Terra Indígena Sangra- douro-Volta Grande, Mato Grosso, Brasil. Cad Saude Publica 2006; 22(9):1865-1872.

8. Vasquez J, Rojas R. Human Rights \& Health - Indigenous People. Pan American Health Organization, 2008. [site da Internet] [acessado 2011 mar 1]. Disponível em: http://new.paho.org/hq/dmdocuments/ 2009/tool\%20box\%2010069_IndigPeople.pdf

9. Rezende F, Rosado L, Franceschinni S, Rosado G, Ribeiro R, Marins JCB. Revisão crítica dos métodos disponíveis para avaliar a composição corporal em grandes estudos populacionais e clínicos. Arch Latinoam. Nutr 2007; 57(4):327-334.

10. International Diabetes Federation. The IDF consensus worldwide deunition of the metabolic syndrome [site na Internet]. [acessado 2011 mar 23]. Disponível em: http://www.pitt.edu/ super1/Metabolic/ IDF1.pdf

11. Taylor RW, Jones IE, Williams SM, Gouldinng A. Body fat percentages measured by dual-energy Xray absorptiometry corresponding to recently recommended body mass index cutoffs for overweight and obesity in children and adolescents aged 3-18 y. Am J Clin Nutr 2002; 76(6):1416-1421.

12. Gallagher D, Heymsfield SB, Heo M, Jebb SA, Murgatroyd PR, Sakamoto Y. Healthy percentage body fat ranges: an approach for developing guidelines based on body mass index1-3. Am J Clin Nutr 2000; 72(3):694-701.

13. Lourenço AEP, Santos RV, Orellana JDY, Coimbra CEA Jr. Nutrition Transition in Amazonia: Obesity and Socioeconomic Change in the Suruí Indians from Brazil. Am J of Hum Biol 2008; 20(5):564-571. 
14. Sampei MA, Canó EM, Fagundes U, Lima EES, Rodrigues D, Sigulem DM, Baruzzi RG. Avaliação antropométrica de adolescentes Kamayurá, povo indígena do Alto Xingu, Brasil Central (2000-2001). Cad Saude Publica 2007; 23(6):1443-1453.

15. Anjos LA. Índice de massa corporal (massa corporal/estatura ${ }^{2}$ ) como indicador do estado nutricional de adultos: revisão de literatura. Rev Saude Publica 1992; 26(6):431-436.

16. Kuczmarski RJ, Flegal KM. Criteria for definition of overweight in transition: background and recommendations for the United States. Am J Clin Nutr 2000; 72(5):1074-1081.

17. Andrade RG, Pereira RA, Sichieri R. Consumo alimentar de adolescentes com e sem sobrepeso do Município do Rio de Janeiro. Cad Saude Publica 2003; 19(5):1485-1495.

18. Ricardo DR, Araújo CGS. Índice de massa corporal: um questionamento baseado em evidências. Arq Bras Cardiol 2002; 79(1):61-69

19. Ellis KJ, Abrams SA, Wong WW : Monitoring childhood obesity: assessment of the weight/Height 2 index. Am J Epidemiol 1999; 150:939-946.

20. Sardinha LB, Going SB, Teixeira PJ, Lohman TG. Receiver operating characteristic analysis of body mass index, triceps skinfold thickness, and arm girth for obesity screening in children and adolescents. Am J Clin Nutr 1999; 70(6):1090-1095.

21. World Health Organization. Child growth standards: growth velocity based on weight, length and head circumference: methods and development. [site da Internet]. [acessado 2011 abr 12]. Disponível em: http://www.who.int/childgrowth/publications/ technical_report_velocity/en/index.html

22. World Health Organization. The use and interpretation of antropometry: report of a WHO expert committee. [site da Internet]. [acessado 2011 abr 16]. Disponível em: http://www.who.int/childgrowth/ publications/physical_status/en/index.html

23. Lipschitz DA. Screening for nutritional status in the elderly. Primary Care 1994: 21(1):55-67.

24. International Diabetes Federation (IDF). The IDF consensus deunition of the metabolic syndrome in children and adolescents. [site na Internet]; [acessado 2011 mar 08]. Disponível em: http://www.idf.org/ metabolic-syndrome/children

25. Almeida LS, Vilela R, Passos F. Xukuru-Kariri: Saúde na Fazenda Canto. Maceió: Edufal; 2005.

26. Parisi RSB. Xukuru Kariri: a reconstituição da trajetória de um grupo indígena remanejado e suas habitações em "novaterra" [tese]. São Carlos: Universidade de São Paulo; 2008.

27. Pan W, Flegal KM, Chang H, Yeh W, Yeh C, Lee W. Body mass index and obesity-related metabolic disorders in Taiwanese and US whites and blacks: implications for definitions of overweight and obesity for Asians. Am J Clin Nutr 2004; 79(1):31-39

28. Woo S, Shin S, Yun YH, Yoo T, Huh B. Cut-off point of BMI and obesity-related comorbidities and mortality in middle-aged. Obes Res 2004; 12(12):20312040
29. Barros DC, Silva DO, Gugelmin SA. Vigilância Alimentar e Nutricional para a Saúde Indígena, Fundação Oswaldo Cruz. Escola Nacional de Saúde Publica Sergio Arouca. Rio de Janeiro: Ed. Fiocruz; 2007.

30. Lourenço AEP. Avaliação do estado nutricional em relação a aspectos socioeconômicos de adultos indígenas Suruí, Rondonia, Brasil [dissertação]. Rio de Janeiro: Fiocruz; 2006. [acessado 2011 maio 18]. Disponível em: http://www.bvsde.paho.org/texcom/ nutricion/zanetti.pdf

31. Corral AR, Somers VK, Sierra-Johnson J, Thomas RJ, Collazo-Clavell ML, Korinek J, Allison TG, Batsis JA, Sert-Kuniyoshi FH, Lopez-Jimenez F. Accuracy of Body Mass index to Diagnose Obesity In the USA Adult population. Int J Obes 2008; 32(6):959966.

32. Cândido APC, Freitas SN, Machado-Coelho GLL. Anthropometric measurements and obesity diagnosis in schoolchildren. Acta Paediatr 2011; 100(9):120-124.

33. Freitas SN, Caiaffa WT, César CC, Cândido APC, Faria VA, Nascimento RM, Machado-Coelho GLL. A comparative study of methods for diagnosis of obesity in an urban mixed-race population in Minas Gerais, Brazil. Public Health Nutr 2007: 10(9):883-890

34. Heyward VH, Stolarczyk LM. Applied Body Composition Assessment. 1a Edição. Illinois: Human Kinetics; 1996.

35. Loftin M, Nichols J, Going S, Sothern M, Schmitz KH, Ring K, Tuuri G, Stevens J. Comparison of the validity of anthropometric and bioelectric impedance equations to assess body composition in adolescent girls. Int J Body Compos Res 2007; 5(1):1-8.

36. Rising R, Swinburn B, Larson K, Ravussin E. Body composition in Pima Indians: validation of bioelectrical resistance. Am J Clin Nutr 1991; 53(3): 594-598.

37. Lohman TG, Caballero B, Himes JH, Davis CE, Stewart D, Houtkooper L, Going SB, Hunsberger S, Weber JL, Reid R, Stephenson L. Estimation of body fat from anthropometry and bioelectrical impedance in Native American children. Int J Obes 2000; 24(8):982-988.

38. Leite MS, Santos RV, Gugelmin SA, Coimbra Júnior CEA. Crescimento físico e perfil nutricional da população indígena Xavante de Sangradouro-Volta Grande, Mato Grosso, Brasil. Cad Saude Publica 2006; 22(2):265-276

39. Bonita R, Beaglehole R, Kjellström T. Epidemiologia básica. 2a Edição. São Paulo: Artes Médicas; 2010. [tradução e revisão científica Juraci A. Cesar]

Artigo apresentado em 31/08/2011

Aprovado em 02/09/2011

Versão final aprovada em 06/11/2011 